\title{
On the diophantine equation $\frac{x^{m}-1}{x-1}=y^{n}$
}

by

LI Yu and MaOHUA LE (Zhanjiang)

1. Introduction. Let $\mathbb{Z}, \mathbb{N}, \mathbb{Q}$ be the sets of integers, positive integers and rational numbers respectively. The solutions $(x, y, m, n)$ of the equation

$$
\frac{x^{m}-1}{x-1}=y^{n}, \quad x, y, m, n \in \mathbb{N}, x>1, y>1, m>2, n>1,
$$

were investigated in many papers. In this respect, Ljunggren [6] proved that equation (1) has only the solutions $(x, y, m, n)=(3,11,5,2)$ and $(7,20,4,2)$ with $n=2$. Shorey and Tijdeman [10, Theorem 12.5] showed that equation (1) has only finitely many solutions $(x, y, m, n)$ if at least one of the following conditions holds: (i) $x$ is fixed, (ii) $m$ is fixed, (iii) $n$ has a fixed prime factor, (iv) $y$ has a fixed prime factor. Moreover, all the solutions can be effectively determined. In general, Shorey and Tijdeman conjectured that equation (1) has only finitely many solutions $(x, y, m, n)$. This problem has not been resolved yet.

Recently, Le [4] proved that if $(x, y, m, n)$ is a solution of (1) such that $x$ is a prime power and $y \equiv 1(\bmod x)$, then $x^{m}<C$, where $C$ is an effectively computable absolute constant. In this paper we improve this result as follows:

TheOREM 1. The equation (1) has no solution $(x, y, m, n)$ with $y \equiv 1$ $(\bmod x)$.

For fixed $a, b \in \mathbb{N}$ with $\operatorname{gcd}(a, b)=1$, we denote by $\operatorname{ord}_{b} a$ the least positive integer value of $t$ for which $a^{t} \equiv 1(\bmod b)$. In [1], Edgar showed that if $(x, y, m, n)$ is a solution of $(1)$, then $m=\operatorname{ord}_{y} x$. Simultaneously, he asked if

$$
n=\operatorname{ord}_{x} y \text {. }
$$

Edgar offered $\$ 50.00$ for settling this problem. On using Theorem 1, we can completely solve this problem as follows:

Supported by the National Natural Science Foundation of China. 
THEOREM 2. Every solution of (1) satisfies (2).

Let $\mathbb{P}$ be the set of odd primes. Edgar asked if there exists a solution $(x, y, m, n)$ of (1) which satisfies $x, y \in \mathbb{P}, m \geq 5$ and $(x, y, m, n) \neq$ $(3,11,5,2)$ (see $[2$, Problem D10]). Shi [8] posed a similar problem for $x=3$. In this paper we prove the following result.

THEOREM 3. If $(x, y, m, n)$ is a solution of (1) with $x \in \mathbb{P}$, then $\operatorname{gcd}(x(x-1), n)=1$. Moreover, the equation (1) has only the solution $(x, y, m, n)=(3,11,5,2)$ with $x=3$.

Let $a, m \in \mathbb{N}$ with $1 \leq a \leq 9$ and $m>1$, and let $N_{a}(m)=a+10 a+$ $\ldots+10^{m-1} a$. Obláth [7] proved that if $a>1$, then $N_{a}(m)$ is never a perfect power. Shorey and Tijdeman $[9$, Theorem 6$]$ proved that $N_{1}(m)$ is not a $p$ th power for any $p \in \mathbb{P}$ with $p \leq 19$. In this respect, we have:

TheOREM 4. $N_{1}(m)$ is never a perfect power.

All the above-mentioned results are in tune with the conjecture of Shorey and Tijdeman.

\section{Proofs of Theorems 1 and 2}

Proof of Theorem 1 . Let $(x, y, m, n)$ be a solution of (1) with $y \equiv$ $1(\bmod x)$. By [6], the only solutions of $(1)$ with $2 \mid n$ are $(x, y, m, n)=$ $(3,11,5,2)$ and $(7,20,4,2)$. We may assume that $2 \nmid n$. Since $n>1, n$ must has an odd prime factor $p$. Then $\left(x, y^{n / p}, m, p\right)$ is a solution of (1) satisfying $y^{n / p} \equiv 1(\bmod x)$ and $p$ is an odd prime. Therefore, it suffices to consider the case that $n$ is an odd prime.

If $n \mid x$ and $n^{\alpha} \| x$, then from $y^{n}-1=x\left(x^{m-2}+\ldots+x+1\right)$ we get $n^{\alpha} \| y^{n}-1$, hence $n^{\alpha-1} \| y-1$, which contradicts $y \equiv 1(\bmod x)$. So we have $n \nmid x$.

If $x-1$ is an $n$th power, then $x-1=y_{1}^{n}$, and $x^{m}-1=\left(y_{1} y\right)^{n}$ for some $y_{1} \in \mathbb{N}$. This is impossible by the proof of [10, Theorem 12.3]. Thus $(x-1)^{1 / n} \notin \mathbb{Q}$.

Let $\theta=(x-1)^{1 / n}$, and let $K=\mathbb{Q}(\theta)$. Then $K$ is an algebraic number field of degree $n$. Let $O_{K}$ and $U_{K}$ be the algebraic integer ring and the unit group of $K$ respectively. For $\alpha_{1}, \ldots, \alpha_{r} \in O_{K}$, let $\left[\alpha_{1}, \ldots, \alpha_{r}\right]$ be the ideal of $K$ generated by $\alpha_{1}, \ldots, \alpha_{r}$, and let $N\left(\left[\alpha_{1}, \ldots, \alpha_{r}\right]\right)$ denote the norm of $\left[\alpha_{1}, \ldots, \alpha_{r}\right]$. Since $\theta=(x-1)^{1 / n}$, we have

$$
[x]=\left[1+\theta^{n}\right]=[1+\theta]\left[\frac{1+\theta^{n}}{1+\theta}\right] .
$$

On the other hand, by (1), we get

$$
[x]^{m}=\left[1+y^{n} \theta^{n}\right]=[1+y \theta]\left[\frac{1+y^{n} \theta^{n}}{1+y \theta}\right] .
$$


Since $y \equiv 1(\bmod x)$, we have

$$
[1+\theta] \mid[1+y \theta]
$$

Further, since $n \nmid x$, the ideals $[1+y \theta]$ and $\left[\left(1+y^{n} \theta^{n}\right) /(1+y \theta)\right]$ are coprime. Therefore, by (4) and (5), we get

$$
[1+\theta]^{m} \mid[1+y \theta]
$$

Notice that $N([1+\theta])=x$ and $N([1+y \theta])=x^{m}$. We find from (6) that $[1+y \theta]=[1+\theta]^{m}$. So we have

$$
1+y \theta=(1+\theta)^{m} \varepsilon, \quad \varepsilon \in U_{K}, N_{K / \mathbb{Q}}(\varepsilon)=1,
$$

where $N_{K / \mathbb{Q}}(\varepsilon)$ is the norm of $\varepsilon$. By the proof of [5, Theorem], (7) is impossible. Thus, the equation $(1)$ has no solution $(x, y, m, n)$ with $y \equiv 1(\bmod x)$. The theorem is proved.

Proof of Theorem 2. Let $k=\operatorname{ord}_{x} y$. If $(x, y, m, n)$ is a solution of (1), then we have $y^{n} \equiv 1(\bmod x)$ and $n \equiv 0(\bmod k)$. Let $n^{\prime}=n / k$ and $y^{\prime}=y^{k}$. If $n^{\prime}>1$, then $\left(x, y^{\prime}, m, n^{\prime}\right)$ is a solution of (1) with $y^{\prime} \equiv 1$ $(\bmod x)$. By Theorem 1 , this is impossible. Thus $n^{\prime}=1$ and the theorem is proved.

3. Proofs of Theorems 3 and 4. Theorems 3 and 4 are based on the following result from [5]:

Lemma. Equation (1) has no solution $(x, y, m, n)$ with $\operatorname{gcd}(x \varphi(x), n)=1$, where $\varphi(x)$ is Euler's totient function of $x$.

Proof of Theorem 3. Since $\varphi(x)=x-1$ for any $x \in \mathbb{P}$, the first part of Theorem 3 is an immediate consequence of the Lemma.

By the Lemma with $x=3$, we get either $2 \mid n$ or $3 \mid n$. By [6], the equation (1) has only the solution $(x, y, m, n)=(3,11,5,2)$ with $x=3$ and $2 \mid n$. In [3], Inkeri showed that equation (1) has only the solution $(x, y, m, n)=$ $(18,7,3,3)$ with $1<x<70$ and $3 \mid n$. Thus the theorem is proved.

Proof of Theorem 4. If $N_{1}(m)$ is a perfect power, then equation (1) has a solution $(x, y, m, n)$ with $x=10$. By the Lemma, we get either $2 \mid n$ or $5 \mid n$. By [9, Theorem 6], this is impossible. The proof is complete.

Acknowledgements. The authors would like to thank the referee for his valuable suggestions.

\section{References}

[1] H. Edgar, Problems and some results concerning the diophantine equation $1+A+$ $A^{2}+\ldots+A^{x-1}=P^{y}$, Rocky Mountain J. Math. 15 (1985), 327-329. 
[2] R. K. Guy, Unsolved Problems in Number Theory, Springer, New York, 1981.

[3] K. Inkeri, On the diophantine equation $a\left(x^{m}-1\right) /(x-1)=y^{n}$, Acta Arith. 21 (1972), 299-311.

[4] M.-H. Le, A note on the diophantine equation $\left(x^{m}-1\right) /(x-1)=y^{n}$, ibid. 64 (1993), 19-28.

[5] -, A note on perfect powers of the form $x^{m-1}+\ldots+x+1$, ibid. 69 (1995), 91-98.

[6] W. Ljunggren, Noen setninger om ubestemte likninger av formen $\left(x^{n}-1\right) /(x-1)$ $=y^{q}$, Norsk Mat. Tidsskr. 25 (1943), 17-20.

[7] R. Obláth, Une propriété des puissances parfaites, Mathesis 65 (1956), 356-364.

[8] W.-J. Shi, On K 4 simple groups, Kexue Tongbao 36 (1991), 1281-1283 (in Chinese).

[9] T. Shorey and R. Tijdeman, New applications of diophantine approximations to diophantine equations, Math. Scand. 39 (1976), 5-18.

[10] —, - Exponential Diophantine Equations, Cambridge Univ. Press, 1986.

DEPARTMENT OF MATHEMATICS

ZHANJIANG TEACHERS' COLLEGE

P.O. BOX 524048

ZHANJIANG, GUANGDONG, P.R. CHINA

Received on 18.7.1994

and in revised (combined) form on 21.4.1995

$(2639 \& 2718)$ 